\title{
Trans-Catheter Aortic Valve Replacement Thrombus in Patient on Xarelto and Aspirin Resolved Three Months After Warfarin
}

\author{
Obiora Maludum ${ }^{\mathrm{a}, \mathrm{c}}$, Firas Ajam ${ }^{\mathrm{b}}$, Anas Alrefaee ${ }^{\mathrm{b}}$, Hetavi Mahida ${ }^{\mathrm{b}}$, Nene Ugoeke ${ }^{\mathrm{a}}$, \\ Dawn Calderon ${ }^{\mathrm{a}}$, Renato Apolito ${ }^{\mathrm{a}}$
}

\begin{abstract}
Trans-catheter aortic valve replacement has been gaining ground since it was performed in 2002. Indications for the procedure are also expanding and are expected to include patients with intermediate risk for perioperative mortality. There are no guidelines on the use of anticoagulation in patients who have undergone trans-catheter aortic valve replacement. We present an interesting case of an 82-year-old gentleman with past medical history of paroxysmal atrial fibrillation with CHA2DS2-VASc (a clinical prediction tool for estimating risk of stroke in patients with atrial fibrillation) score of 3 , coronary artery disease, pernicious anemia, and severe symptomatic aortic stenosis who had trans-catheter aortic valve replacement. He was on Xarelto and aspirin, and still developed thrombus on the aortic valve many months after the procedure. The thrombus resolved after few months of anti-coagulation with Coumadin. This case shows that thrombus on the valve can still occur post-procedure even in patients who are on direct oral anticoagulant and aspirin. This calls into question if there is need to anti-coagulate all patients after TAVR procedure with Coumadin for at least 3 - 6 months. As our knowledge of the post-TAVR complications and outcomes continues to grow, we may be able to answer this question in the future, especially with large scale research studies.
\end{abstract}

Keywords: TAVR; Aortic prosthetic valve; Thrombosis; Anticoagulation

\section{Introduction}

The first trans-catheter aortic valve replacement (TAVR) procedure was performed in 2002 [1]. Since then, it has become

Manuscript submitted May 7, 2018, accepted June 20, 2018

${ }^{a}$ Cardiovascular Disease Section, Department of Internal Medicine, Jersey Shore University Medical Center, Neptune, NJ, USA

bDepartment of Internal Medicine, Jersey Shore University Medical Center, Neptune, NJ, USA

${ }^{\mathrm{c} C o r r e s p o n d i n g ~ A u t h o r: ~ O b i o r a ~ M a l u d u m, ~ C a r d i o v a s c u l a r ~ D i s e a s e ~ S e c t i o n, ~}$ Department of Internal Medicine, Jersey Shore University Medical Center, Neptune, NJ 07753, USA. Email: Obimal@yahoo.com

doi: https://doi.org/10.14740/jmc3084w the standard of care in prohibitive and high risk patients for surgical aortic valve replacement [2]. Since the first US Food and Drug Administration approval in 2011, over 50,000 patients have undergone TAVR in the United States alone [2]. Although clinical or symptomatic leaflet thrombosis is uncommon, it is a serious adverse event following TAVR with a grave prognosis.

As indications for TAVR is expected to expand in the future to include patients at intermediate and perhaps low risk for perioperative mortality following surgery, an early diagnosis of leaflet thrombosis and treatment with antithrombotic therapy would be critical to optimize clinical outcomes.

It was recorded that most cases appear to occur in the first year following TAVR, and the role of direct oral anticoagulants (DOAC) is being investigated in ongoing trials [3]. Accordingly, there is uncertainty on how to prevent and treat this complication in patients who present after 1 year while on DOAC.

\section{Case Report}

Our patient is an 82-year-old gentleman with a past medical history of paroxysmal atrial fibrillation with a CHA2DS2VASc score of 3, coronary artery disease, pernicious anemia, and severe symptomatic aortic stenosis. Pre-procedural transthoracic echocardiography (TTE) showed an aortic valve area of $0.67 \mathrm{~cm}^{2}$ and preserved left ventricular function. A $29 \mathrm{~mm}$ Edwards Sapien III valve was successfully deployed without post dilatation. The post-procedural mean trans-valve gradient was $5 \mathrm{~mm} \mathrm{Hg}$, and no significant para-valvular regurgitation was observed by transesophageal echocardiogram (TEE). The patient opted for off-label Xarelto for anticoagulation because he was not doing well on warfarin, with very labile international normalized ratio (INR). A long discussion about the off-label use of Xarelto in the setting of bio-prosthetic aortic valve replacement led to his decision to pursue this along with aspirin for paroxysmal atrial fibrillation, stroke prophylaxis. He was then discharged on Xarelto $20 \mathrm{mg}$ daily and aspirin 81 mg daily. He was otherwise doing well, and without dyspnea or chest tightness. On routine outpatient follow-up, we noted a murmur that was increased in intensity and repeat TTE showed that the gradients had increased to a mean gradient of $24 \mathrm{~mm}$ $\mathrm{Hg}$. There was thickening of the aortic valve and thrombosis was concerning and suspected. There was no fever, chills, no 

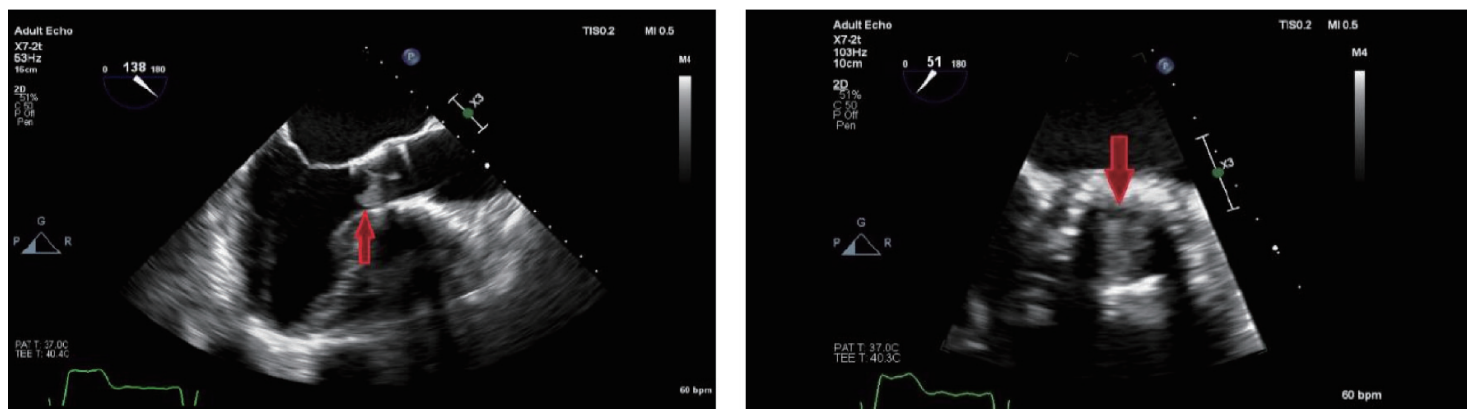

Figure 1. Echocardiographic pictures of THV before treatment. Red arrows point to the thrombus on THV in two different views.

rash, no weight loss, weight gain, or signs of endocarditis or infection, no tooth aches etc. The patient denied any nausea, vomiting, diarrhea. He did undergo TEE which confirmed right coronary cusp thrombus measuring about $1.43 \mathrm{~cm}$ by $0.91 \mathrm{~cm}$, which was layered, non-mobile, with normal valve function except for mild restriction leaflets (Fig.1).

It was decided to admit him for high intensity IV heparin with bridge to warfarin and goal of INR between 2.5 and 3.5. He was discharged from the hospital few days later when his INR reached goal. Repeat TEE 3 months later showed complete resolution of the thrombus and normal valve function (Fig. 2).

\section{Discussion}

In a recent large consecutive cohort of 405 patients having TAVR performed with the Edwards balloon-expandable transcatheter heart valve (THV), the thrombus was diagnosed 1 - 3 months after the procedure by multi-detector computed tomography (MDCT) in 7\% of the patients [4].

Based on previous studies, tt has been noticed that the occurrence of THV thrombosis was associated with large THV size and in cases in which warfarin was not used as an option for antithrombotic agent [4-8]. THV thrombosis is expected to occur in the early period after TAVR and has not previously been reported later than 7 months post-TAVR [4, 9]. This case showed that THV dysfunction or an increase in murmur intensity should evoke prompt investigation for possible thrombus formation.

The predisposing factors for TAVR thrombosis in our case were the presence of paroxysmal atrial fibrillation, the use of Xarelto instead of warfarin and the use of mono antiplatelet therapy.

In a recent meta-analysis that compared aspirin versus aspirin and clopidogrel following TAVR, there was no difference in the 30-day clinical and cerebrovascular adverse event rates; however, a trend toward more bleeding in the aspirin and clopidogrel group was demonstrated, also it was recorded that monotherapy with aspirin was associated with a THV thrombosis risk of $25 \%$, and importantly, all patients who experienced obstructive THV thrombosis received antiplatelet therapy alone [10].

Our patient did not experience any kind of cardiovascular symptoms. The thrombosis was discovered on a regular follow-up at the office with his cardiologist, which stresses the importance of regular long-term follow-up and raises the question of how frequently and for how long should those patients receive echocardiographic assessment of their valves. Based on a study that was done among 460 consecutive patients who underwent TAVR with the Edwards Sapien XT or Sapien 3 (Edwards Lifesciences, Irvine, California), THV thrombosis occurred in 28 of $405(7 \%)$ patients. A total of 23 patients had subclinical THV thrombosis, whereas five (18\%) patients experienced clinically overt obstructive THV thrombosis [4].

There are no clear guidelines on how to treat THV thrombus or the choice of anticoagulant for prevention. We and others have observed incidence of THV thrombosis in patients who are not on warfarin, thus indicating that short-term warfarin treatment or even long-term NOAC anticoagulants treatment of THV thrombosis might not be sufficient in patients
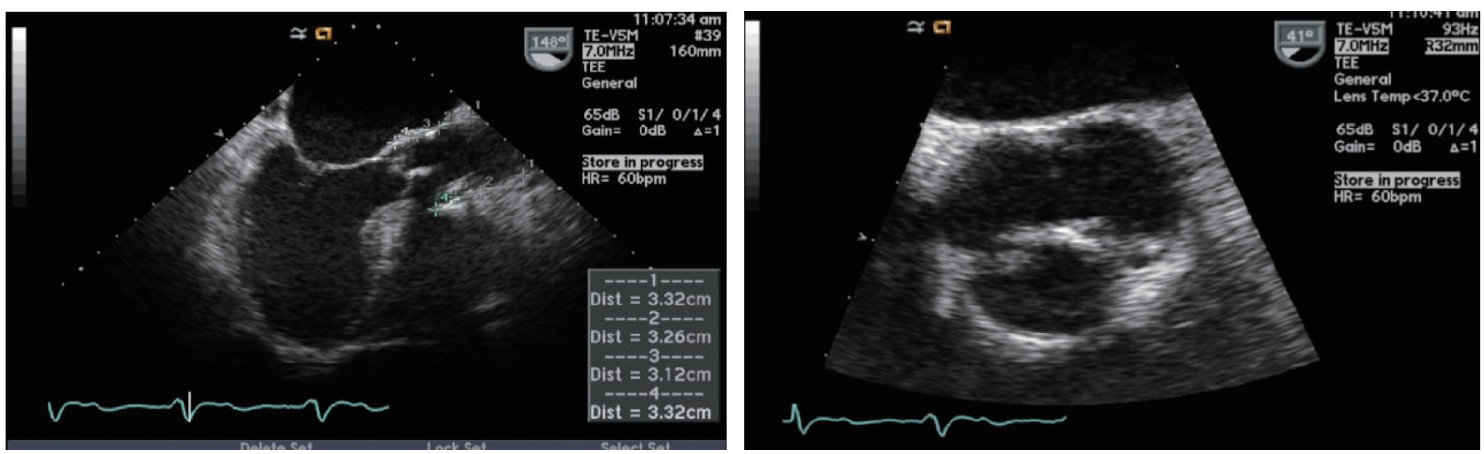

Figure 2. Echocardiographic pictures of the same patient showing resolution of the thrombus after treatment with warfarin. 
who are prone to developing THV thrombosis [6].

\section{Conclusions}

THV thrombosis may develop late after TAVR despite treatment with DOAC and aspirin, and can be reversed with warfarin. As our knowledge of the outcomes post-TAVR continues to increase, this may lead to development of guideline in the future about the use of anticoagulation, especially warfarin, and for how long following the implantation of the valve. Large scale randomized study may be needed in the future to guide the choice of anticoagulant and duration of treatment.

\section{Conflict of Interest}

No conflict of interest from any of the authors.

\section{Refrences}

1. Cribier A, Eltchaninoff H, Bash A, Borenstein N, Tron C, Bauer F, Derumeaux G, et al. Percutaneous transcatheter implantation of an aortic valve prosthesis for calcific aortic stenosis: first human case description. Circulation. 2002;106(24):3006-3008.

2. Otto CM, Kumbhani DJ, Alexander KP, Calhoon JH, Desai MY, Kaul S, Lee JC, et al. 2017 ACC Expert consensus decision pathway for transcatheter aortic valve replacement in the management of adults with aortic stenosis: a report of the american college of cardiology task force on clinical expert consensus documents. J Am Coll Cardiol. 2017;69(10):1313-1346.
3. Windecker S, Tijssen J, Giustino G, Guimaraes $A H$, Mehran R, Valgimigli M, Vranckx P, et al. Trial design: Rivaroxaban for the prevention of major cardiovascular events after transcatheter aortic valve replacement: Rationale and design of the GALILEO study. Am Heart J. 2017; 184:81-87.

4. Hansson NC, Grove EL, Andersen HR, Leipsic J, Mathiassen ON, Jensen JM, Jensen KT, et al. Transcatheter Aortic Valve Thrombosis: Incidence, Predisposing Factors, and Clinical Implications. J Am Coll Cardiol. 2016;68(19):2059-2069.

5. Pache G, Schoechlin S, Blanke P, Dorfs S, Jander N, Arepalli CD, Gick M, et al. Early hypo-attenuated leaflet thickening in balloon-expandable transcatheter aortic heart valves. Eur Heart J. 2016;37(28):2263-2271.

6. Makkar RR, Fontana G, Jilaihawi H, Chakravarty T, Kofoed KF, De Backer O, Asch FM, et al. Possible subclinical leaflet thrombosis in bioprosthetic aortic valves. N Engl J Med. 2015;373(21):2015-2024.

7. Latib A, Naganuma T, Abdel-Wahab M, Danenberg H, Cota L, Barbanti M, Baumgartner H, et al. Treatment and clinical outcomes of transcatheter heart valve thrombosis. Circ Cardiovasc Interv. 2015;8(4):e001779.

8. Mylotte D, Andalib A, Theriault-Lauzier P, Dorfmeister M, Girgis M, Alharbi W, Chetrit M, et al. Transcatheter heart valve failure: a systematic review. Eur Heart J. 2015;36(21):1306-1327.

9. Pingpoh C, Pache G, Nawras D, Guenkel L, Sami K, Zeh W, Zimmer E, et al. Valve thrombosis 7 months after transcatheter aortic valve implantation. Ann Thorac Surg. 2014;98(3):1079-1081.

10. Hassell ME, Hildick-Smith D, Durand E, Kikkert WJ, Wiegerinck EM, Stabile E, Ussia GP, et al. Antiplatelet therapy following transcatheter aortic valve implantation. Heart. 2015;101(14):1118-1125. 